\title{
Scaling in a network model of a multispecies ecosystem
}

\author{
Ricard V. Soléa,b,*, David Alonso ${ }^{\mathrm{a}, \mathrm{c}}$, Alan McKane ${ }^{\mathrm{d}}$
}

${ }^{a}$ Complex Systems Research Group, Department of Physics, FEN, Universitat Politècnica de Catalunya, Sor Eulalia d' Anzizu s/n, Campus Nord B4, 08034 Barcelona, Spain

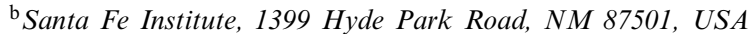

${ }^{c}$ Department of Ecology, Facultat de Biologia, Universitat de Barcelona, Diagonal 645, 08045 Barcelona, Spain

${ }^{\mathrm{d}}$ Department of Theoretical Physics, University of Manchester, Manchester M13 9PL, UK

Received 20 January 2000

\begin{abstract}
A new model ecosystem of many interacting species is introduced in which the species are connected through a random matrix with a given connectivity. The model is studied both analytically and by numerical simulations. A probability distribution derived from the model is in good agreement with simulations and field data. It is also shown that the connectivity, $C$, and the number of species, $S$, are linked through the scaling relation $\langle S\rangle=k(C)^{-1+\varepsilon}$, which is observed in real ecosystems. Our approach suggests a natural link between log-normal and power-law distributions of species abundances. (c) 2000 Elsevier Science B.V. All rights reserved.
\end{abstract}

PACS: 87.10.+e; 05.40.+j; 05.45.+b

Keywords: Ecosystem; Stochastic dynamics; Random networks; Multispecies communities; Master equation

The universal properties of complex biosystems have attracted the attention of physicists over the last decade or so. The standard approach to complex ecosystems is based on the classical Lotka-Volterra (LV) $S$-species model [1]:

$$
\frac{\mathrm{d} N_{i}}{\mathrm{~d} t}=N_{i}\left(\varepsilon_{i}-\sum_{j=1}^{S} \alpha_{i j} N_{j}(t)\right),
$$

where $\left\{N_{i}\right\}(i=1, \ldots, S)$ is the population size of each species. Here $\varepsilon_{i}$ and $\alpha_{i j}$ are constants that introduce feedback loops and interactions among different species. The

\footnotetext{
* Corresponding author. Fax: +34-93-401-7100.

E-mail address: ricard@complex.upc.es (R.V. Solé).
} 
matrix $\mathbf{A}=\left(\alpha_{i j}\right)$ describes the interaction graph [1] and even a small degree of asymmetry leads to very complicated dynamics, although several generic properties have been identified. In this respect, recent studies on a related class of replicator equations, where an initial random graph evolves towards a highly non-random network revealed remarkable self-organized features [2].

A classical result on randomly wired ecosystems [3] shows that a critical limit to the number of species $S$ exists for a given connectivity $C$. Here $C$ is the fraction of non-zero matrix elements. This limit is sharp (a phase transition): below the critical value the system is stable but it becomes unstable otherwise. The basic inequality is that the community will be stable if $C \leqslant \sigma / S$, where $\sigma$ is a given constant. Specifically, if $P(S, C, \sigma)$ is the probability that the system is stable for a given $(S, C, \sigma)$ set, where $\sigma$ is the matrix variance, then (for large $S$ ) $P(S, C, \sigma) \rightarrow 1$ if $C S \leqslant \sigma$ and $P(S, C, \sigma) \rightarrow 0$ otherwise. Here the equality defines the boundaries between the stable and the unstable domains. Field studies show that, in general, $C=\sigma S^{-1+\varepsilon}$ with $\varepsilon \in[0,1 / 2][4]$.

More recently, some authors have suggested that complex biosystems could be self-organized into a critical state [5,6]. Most of these approaches based on selforganized criticality (SOC), such as the Bak-Sneppen model [5], involve a time scale that is assumed to be very large. But the species properties of a given ecosystem do not change appreciably on a smaller time scale [7] and several field observations suggest that real ecosystems could display some features characteristic of slowly driven interaction dominated threshold systems, as Jensen [8] calls the systems that exhibit SOC-dynamics. In particular:

(i) The analysis of time series [9] shows that the largest Lyapunov exponent is typically close to zero.

(ii) Studies on colonization in islands show that after a critical number of species is reached, extinction events are triggered, following a power-law distribution [10].

(iii) The lifetime distribution of species (defined in terms of local extinction in given areas) is also a power law $N(T) \approx T^{-\theta}$ with $\theta=1.12$ [10] to $\theta=1.6$ [11].

(iv) In species-rich ecosystems, the number of species $N_{s}(n)$ with $n$ individuals is also a power law $N_{s}(n) \approx n^{-\gamma}$ where $\gamma$ is close to one, although log-normal distributions with long tails are also well known [12]. Some recent extensive field data analysis from ecosystems has shown that these communities follow a very well-defined power law in species abundances: $N_{s}(n) \approx n^{-\gamma}$ with $\gamma \in(1,1.25)$ spanning 3-4 decades (S. Pueyo, unpublished data).

Although theoretical explanations for some of these isolated observations have been derived in previous studies (together with the $C-S$ law) they have not been explained within a single theoretical framework.

In this paper, we present a new model which can be used as a simple alternative to the LV formulation and follows the simple (but somewhat different) approach of other studies on random graphs [2]. The model involves a set $\Theta_{s}$ of $S$ (possible) species and a population size $N$. Individuals of different species interact through a matrix $\boldsymbol{\Omega}$, with a pre-defined connectivity $C_{\pi} \in[0,1]$. Our approach is close to previous 


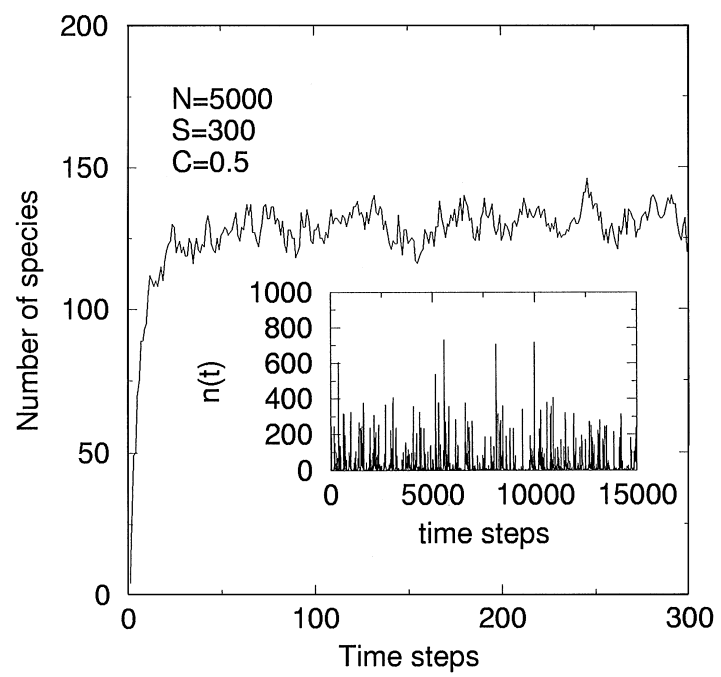

Fig. 1. An example of the time evolution of a single species (inset) from a population of $N=5000$ individuals and $S=300$ species in the pool. Here $C=0.5$ and $\mu=5 \times 10^{-3}$. We can see a wide spectrum of fluctuations in $n(t)$. Main figure: the number of species present in the simulated ecosystem increases up to an average value $\langle S\rangle \approx 125$ imposed by the matrix connectivity.

lattice models of population dynamics where different species can "invade" lattice points already occupied by another species if a previously defined set of rules allows it.

The matrix elements, $\Omega_{i j}$, are randomly chosen from a uniform distribution $\rho(x)$. Each time step, two individuals are taken at random belonging, say, to species $i$ and $j$, respectively. Then if $\Omega_{i j}>\Omega_{j i}$ the individual belonging to $j$ is replaced, with probability $1-\mu$, by a new individual of species $i$. If $\Omega_{i j}=\Omega_{j i}=0$ then nothing happens. This rule is repeated $N$ times and these $N$ updates define our time step. Additionally, with probability $\mu$, any individual can be replaced by another individual of any of the species from $\Theta_{s}$. These rules makes our model close to Eq. (1) when $\alpha_{i j}>0$ ( $S$-species competition model [1]). So two basic reactions are allowed: (i) interaction through $\boldsymbol{\Omega}$ and (ii) random replacement by a new species from the $\Theta_{s}$ pool. The second rule introduces immigration (at a rate $\mu$ ): our driving force [13,14].

An example of the temporal dynamics of this model is shown in Fig. 1, where we can see a wide spectrum of fluctuation sizes, not very different from those observed in some natural communities $[1,9,11,15]$. Starting from any given initial number of species, this model evolves towards a steady-state characterized by a limited subset of species (with average $\langle S\rangle$ ) [16]. The specific subset itself changes in time. Let $C^{*}$ be the probability of having two species connected. Then $C^{*}$ and $\langle S\rangle$ are linked by a power law $\langle S\rangle=k\left(C^{*}\right)^{-1+\varepsilon(\mu)}$. Once this $\langle S\rangle$ is reached, complex fluctuations can arise and under some conditions (see below) power laws are observable. The analysis of the population fluctuations typically shows, for small driving $\mu$ and large enough $C_{\pi}$, a power law $P(n) \approx n^{-\gamma} f_{\mu}(n)$. Here $\gamma=1$ when $N \gg S \gg 1$ and $f_{\mu}(n)$ is an exponential cut-off. The lifetime distribution of species is also a power law $N(T) \approx T^{-\theta}$ and 
for $N \gg S, \theta \in(1,3 / 2)$. The $\theta=3 / 2$ limit corresponds to low-level connectivities (i.e., random-walk-like behavior) and $\theta=1$ to the situation where interactions dominate over immigration. It is remarkable that these limits in the lifetimes correspond to those reported from field studies [10,11].

The limiting case $\mu \rightarrow 0$ shows the exact hyperbolic relation and can be derived from a mean-field approach. It is not difficult to see that the number of species will change in time following:

$$
\frac{\mathrm{d} S}{\mathrm{~d} t}=\mu(N-S)-C^{*} S
$$

where the first term on the right-hand side stands for the continuous introduction of new species at a rate $\mu$ (with an upper limit at $N$ ) and the second for the instability derived from interactions. The previous equation gives an exponential approach to the critical state $S(t)=\left[\mu N-(\mu N-\eta S(0)) \mathrm{e}^{-\eta t}\right] / \eta$ where $\eta \equiv \mu+C^{*}$. This leads at the steady state to

$$
S^{*}=\frac{\mu N}{\mu+C^{*}}
$$

and shows a hyperbolic dependence with $C$ at low immigration rates.

A mean field theory of species abundance can be derived. Let us start with the master equation for this model. If $P(n, t)$ is the probability (for any species) of having $n$ individuals at time $t$, the one-step process is described by [17]

$$
\frac{\mathrm{d} P(n, t)}{\mathrm{d} t}=r_{n+1} P(n+1, t)+g_{n-1} P(n-1, t)-\left(r_{n}+g_{n}\right) P(n, t),
$$

where the one-step transition rates $r_{n} \equiv W(n-1 \mid n)$ and $g_{n} \equiv W(n+1 \mid n)$ are

$$
\begin{aligned}
& r_{n}=C^{*}(1-\mu) \frac{n}{N} \frac{N-n}{N-1}+\frac{\mu}{S}(S-1) \frac{n}{N}, \\
& g_{n}=C^{*}(1-\mu) \frac{n}{N} \frac{N-n}{N-1}+\frac{\mu}{S}\left(1-\frac{n}{N}\right) .
\end{aligned}
$$

From (5) and (6) we see that $r_{0}=0$ and $g_{N}=0$ and we define $g_{-1}=0$ and $r_{N+1}=0$. The stationary distribution $P_{s}(n)$ is obtained from $\mathrm{d} P(n) / \mathrm{d} t=0$ using standard methods [17] namely by writing

$$
P_{s}(n)=\frac{g_{n-1} g_{n-2} \cdots g_{0}}{r_{n} r_{n-1} \cdots r_{1}} P_{s}(0)
$$

and using the normalization condition $\sum_{n=0}^{N} P_{S}(n)=P_{S}(0)+\sum_{n>0} P_{S}(n)=1$, to show that

$$
\left(P_{s}(0)\right)^{-1}=\sum_{n=0}^{N}\left(\begin{array}{l}
N \\
n
\end{array}\right)(-1)^{n} \frac{\Gamma\left(n+\lambda^{*}\right)}{\Gamma\left(\lambda^{*}\right)} \frac{\Gamma\left(1-v^{*}\right)}{\Gamma\left(n+1-v^{*}\right)} .
$$

Here $\lambda^{*}=\mu^{*}(N-1)$ and $v^{*}=N+\mu^{*}(N-1)(S-1)$, where $\mu^{*}=\mu /\left[(1-\mu) S C^{*}\right]$. This sum takes the form of a Jacobi polynomial $P_{N}^{(\alpha, \beta)}(x)[18]$, with $\alpha=-v^{*}, \beta=\lambda^{*}+v^{*}-(N+1)$ and $x=-1$, which can itself be expressed in terms of gamma functions for this value 


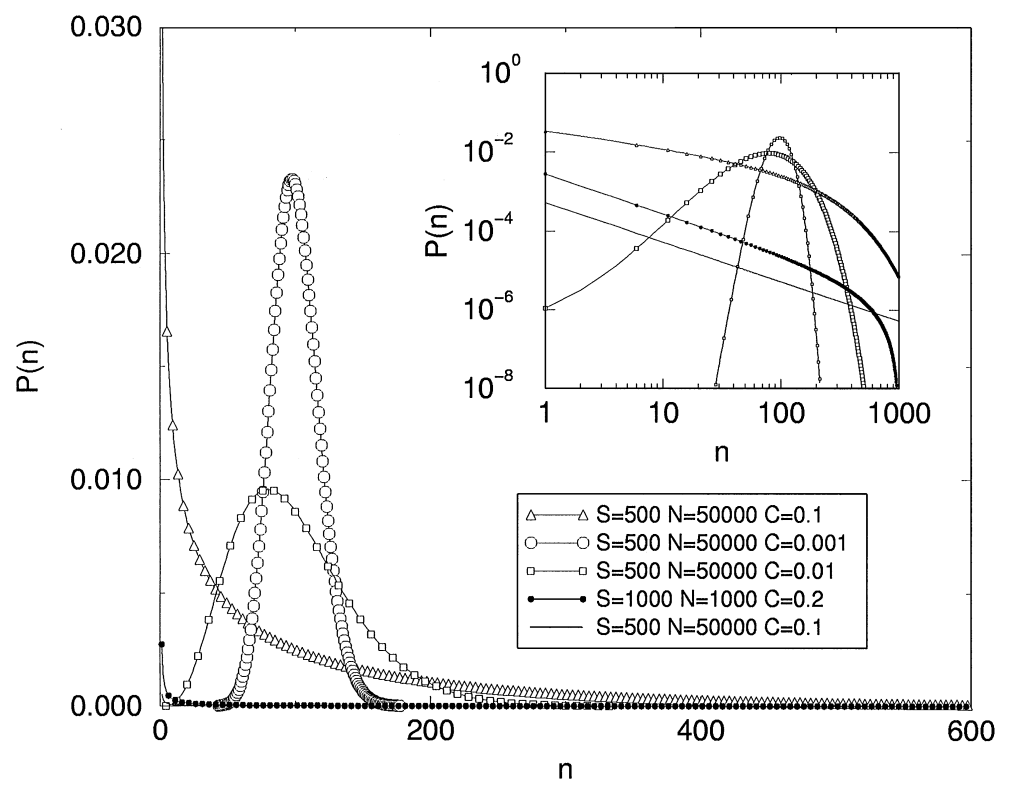

Fig. 2. Stationary probability distribution $P_{S}(n)$ obtained from (4)-(6), with $\mu=10^{-3}$. For different values of $S / N$ and $C$ we get different stationary distributions. For $S=500, N=50,000$ and different connectivities, we get Gaussian $(C=0.001)$, $\log$-normal $(C=0.01)$ or power laws $(C=0.1$, here with a strong bending). As the connectivity or $S / N$ increases, power laws are obtained (black circles).

of $x$. Therefore, using $\beta(p, q)=\Gamma(p) \Gamma(q) / \Gamma(p+q)$, the stationary, normalized solution can be written in the form

$$
P_{S}(n)=\left(\begin{array}{c}
N \\
n
\end{array}\right) \frac{\beta\left(n+\lambda^{*}, v^{*}-n\right)}{\beta\left(\lambda^{*}, v^{*}-N\right)} .
$$

As the connectivity (or the immigration rate) increases, the distributions changes from Gaussian to log-normal and to power laws (Fig. 2). This is expected since very small connectivities will make interactions irrelevant, with fluctuations dominated by random events. We can simplify (9) in the region of interest: $N$ and $S$ large, and $N \gg n_{\max }$, where $n=1, \ldots, n_{\max }$. If in addition $\lambda \ll 1$, which corresponds to $\mu$ small: $\mu \ll S C^{*} / N$, we get a scaling relation $[19,20]$ :

$$
P_{s}(n)=\mathscr{K} n^{-1} \exp \left(-n \mu^{*} S\right),
$$

where $\mathscr{K}=\left(\mu^{*} S\right)^{\lambda^{*}} / \Gamma\left(\lambda^{*}\right)$ in agreement with simulations and field data. These results suggest a simple link between a range of statistical distributions from those generated by multiplicative events to those related with SOC dynamics [21].

From this solution we can compute the stationary number of species $\langle S\rangle=(1-P(0)) S$ and find the relation between this quantity and the probability of having two species connected, $C^{*}$. Under the same conditions that led to $(10)$, we find ${ }^{1}$ that $P_{S}(0)=\left(\mu^{*} S\right)^{\lambda^{*}}$,

\footnotetext{
${ }^{1}$ Since $P_{S}(0)=(S-1) / S$, for $\lambda^{*}$ approaching zero $P_{S}(0)=\left(\mu^{*} S\right)^{\lambda^{*}}$, should be approximated by $P_{S}(0)=$ $((S-1) / S)\left(\mu^{*} S\right)^{\lambda^{*}}$. Then, instead of $(11)$, a correction is needed when $\lambda^{*}$ is tending to zero: $\langle S\rangle-1=$ $\mathscr{A}\left[C^{*}\right]^{-1+\varepsilon(\mu)}$. As $\mu$ is tending to zero, $\langle S\rangle=1$, for high connectivities.
} 


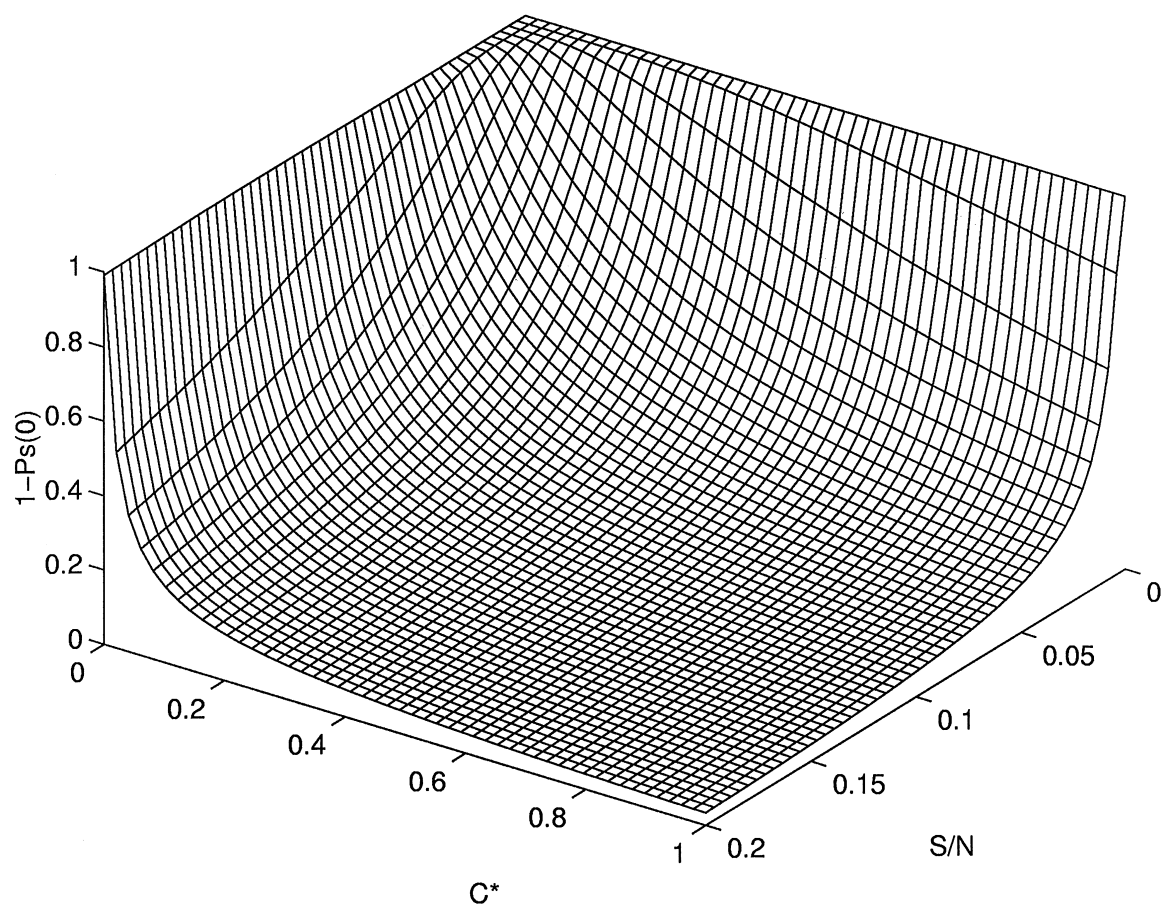

Fig. 3. Fraction of remaining species at steady state as a function of $S / N$ and connectivity $(N=50,000$, $\mu=0.001)$. In this parameter range, hyperbolic-like behavior can be observed for high values of $S / N$.

which in this parameter range gives $\langle S\rangle=S \mu^{*} N \ln \left(1 /\left(\mu^{*} S\right)\right.$. Substituting in the expression for $\mu^{*}$ gives

$$
\begin{aligned}
\langle S\rangle & =\frac{\mu N}{(1-\mu) C^{*}}\left[\ln \left(\frac{1-\mu}{\mu}\right)+\ln C^{*}\right] \\
& \approx \mathscr{A}\left(C^{*}\right)^{-1+\varepsilon(\mu)},
\end{aligned}
$$

where $\varepsilon$ is given by $\varepsilon^{-1}=\ln ((1-\mu) / \mu) \approx \ln \mu^{-1}$ and $\mathscr{A}$ is a constant equal to $N \varepsilon^{-1} \exp \left(-\varepsilon^{-1}\right)$. The additional conditions under which (11) hold are $\lambda^{*} \ll \varepsilon$ and $\left|\ln \left(C^{*}\right)\right| \ll|\ln (\varepsilon)|$. The relation between $\langle S\rangle$ and $C$ is shown in Fig. 3 for different $S / N$ values and a quite large immigration rate $(\mu=0.001)$, and in Fig. 4 for different, but smaller, values of $\mu$. In the last case, the hyperbolic relation (i.e., $\langle S\rangle \approx C^{-1+\varepsilon}$ ) is compared with the exact solution $(\langle S\rangle=(1-P(0)) S)$. In $(11), C^{*}$ appears instead of the fraction of non-zero elements of the connectivity matrix, $C_{\pi}$. Both connectivities have exactly the same meaning, i.e., the probability of having two species connected, as long as completely asymmetrical interaction between species is forbidden. If $\Omega_{i j}$ (the effect of $j$ on $i$ species) is non-zero, then $\Omega_{j i}$ may be extremely low but different from zero. The former assumption is very realistic in natural communities. 

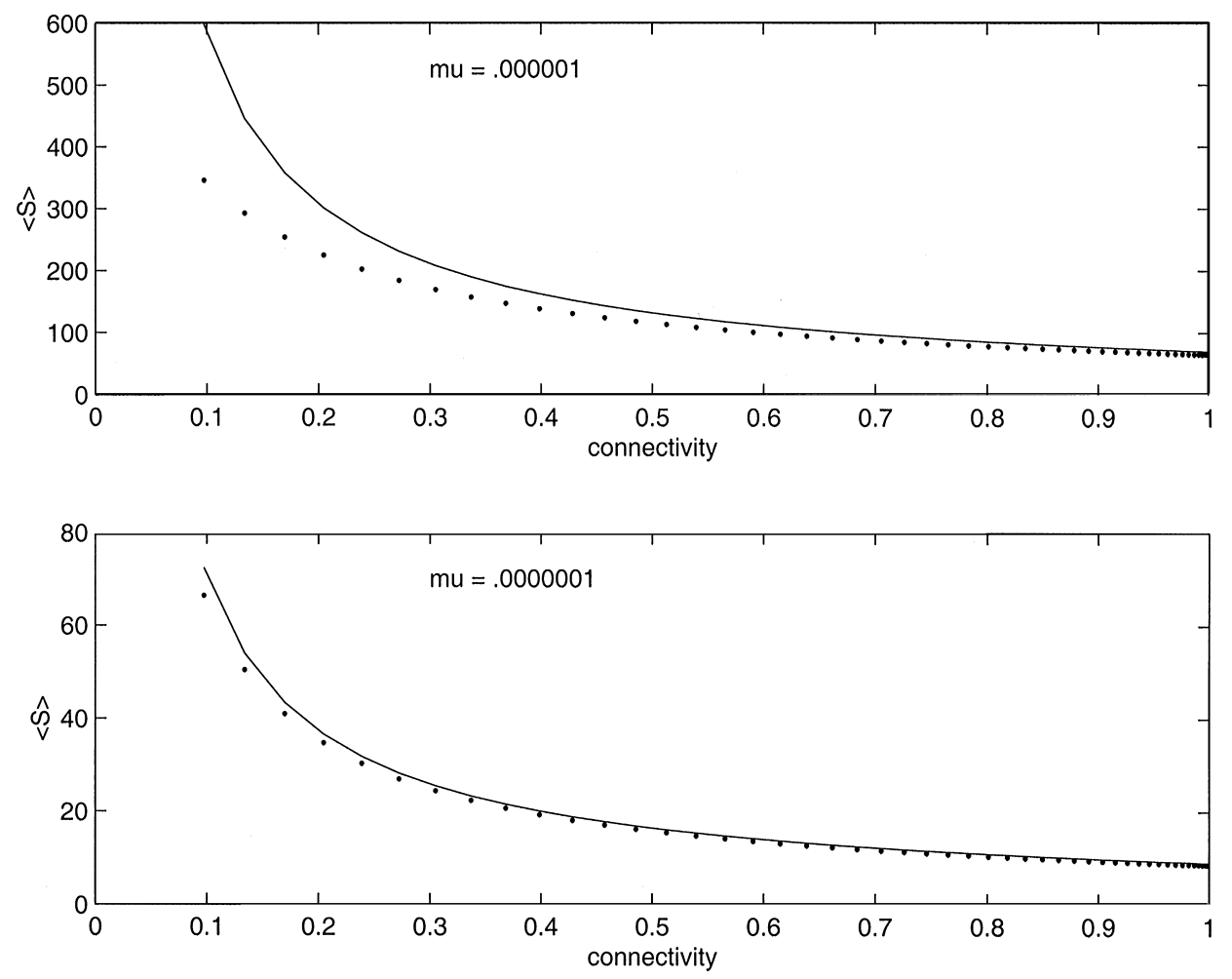

Fig. 4. Scaling between number of species and connectivity: here two different plots are shown, for $N=5 \times 10^{6}, S=500$ and for different values of $\mu$. Dots and solid lines indicate the exact solution $(\langle S\rangle=(1-P(0)) S)$ and the approximation (11), respectively.

In summary, a simple $S$-species network model that shows both log-normal (even Gaussian) distributions as well as critical dynamics has been introduced. Starting from random initial conditions, the system evolves towards a state characterized by some well-defined scaling laws and statistical features. High immigration, small $S / N$ relations or low connectivities lead to log-normal distributions. These are replaced by well-defined power laws as the interactions become more relevant. In the last case, a multiplicity of metastable states, the presence of a threshold in the number of species $S_{c}=\langle S\rangle$, the scale separation between the driving when $\mu$ is small and the system response, fully describe our system as a slowly driven, interaction dominated, threshold SOC-system.

As a consequence of the critical dynamics several well-known field observations are recovered. The interpretation of this model provides a new framework for understanding how complex dynamics emerges in multispecies ecosystems and suggest that some well-known time series from natural communities are the result of critical, instead of deterministic chaotic, behavior. 


\section{Acknowledgements}

The authors thank H. Jensen, R. Engelhardt, S. Pimm, S. Jain, S. Kauffman and K. Sneppen for useful information and discussions. Special thanks to Per Bak for encouraging comments. DA thanks J.P.G. Gamarra for his constant support and his interesting comments. This work has been supported by a grant PB97-0693 and by the Santa Fe Institute (RVS), by a CIRIT grant 1999FI 00524 UPC APMARN (DA) and by EPSRC grant GR/K79307 and the British Council (AM).

\section{References}

[1] R.M. May, Stability and Complexity in Model Ecosystems, Princeton University Press, Princeton, NJ, 1973.

[2] S. Jain, S. Krishna, Phys. Rev. Lett. 81 (1998) 5684.

[3] R.M. May, Nature 238 (1972) 413.

[4] S.L. Pimm, The Balance of Nature, University of Chicago Press, Chicago, 1994.

[5] P. Bak, K. Sneppen, Phys. Rev. Lett. 71 (1993) 4083.

[6] R.V. Solé, S.C. Manrubia, Phys. Rev. E 54 (1996) R42.

[7] J. Maynard Smith, N.C. Stenseth, Evolution 38 (1984) 870.

[8] H. Jensen, Self-Organized Criticality, Cambridge University Press, Cambridge, 1998.

[9] S. Ellner, P. Turchin, Am. Nat. 145 (1995) 343.

[10] T.H. Keitt, P.A. Marquet, J. Theor. Biol. 182 (1996) 161.

[11] T.H. Keitt, H.E. Stanley, Nature 393 (1998) 257.

[12] F.W. Preston, Ecology 43 (185) (1962) 410.

[13] R.V. Solé, D. Alonso, Adv. Complex Systems 1 (1998) 203.

[14] M. Tokeshi, Species Coexistence: Ecological and Evolutionary Perspectives, Blackwell, Oxford, 1999.

[15] A. Ariño, S.L. Pimm, Evol. Ecol. 9 (1995) 429.

[16] S.P. Hubbell, Coral Reefs 16 (Suppl.) (1997) S9.

[17] N.G. Van Kampen, Stochastic Processes in Physics and Chemistry, Elsevier, Amsterdam, 1981.

[18] M. Abramovitz, A. Stegun (Eds.), Handbook of Mathematical Functions, Dover, New York, 1965.

[19] D.G. Kendall, Biometrika 35 (1948) 6.

[20] R.A. Fisher, A.S. Corbet, C.B. Williams, J. Anim. Ecol. 12 (1943) 42.

[21] S. Engen, R. Lande, J. Theor. Biol. 178 (1996) 325. 